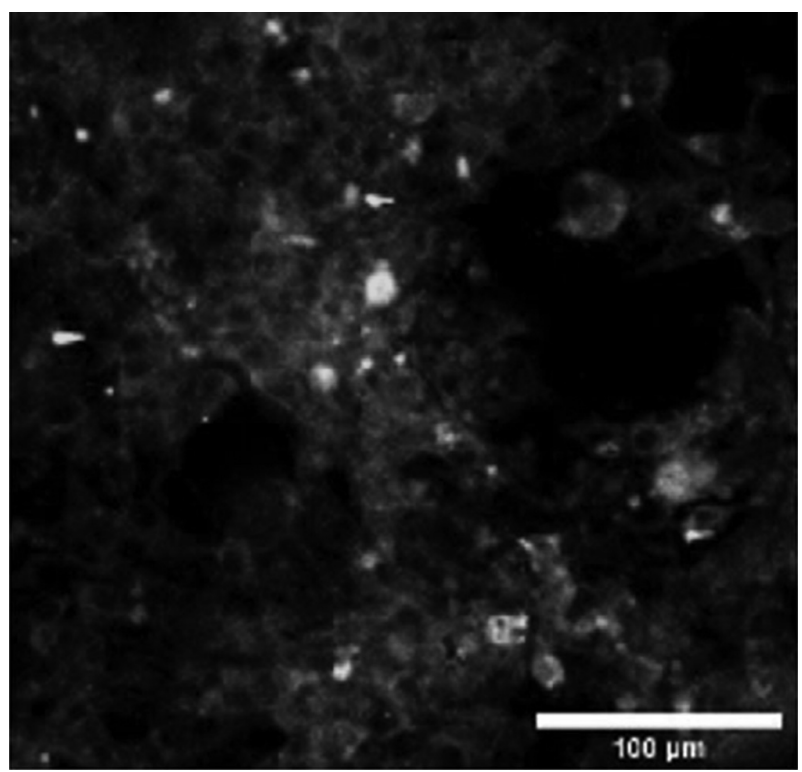

Abstract BS49 Figure 2 Representative image of anti-apelin receptor (green) staining in beating hESC-derived cardiomyocytes. Blue = DAPI, scale bar $=100 \mu \mathrm{m}$

beating hESC-derived cardiomyocytes and we propose to generate hESC-derived phenotypic models by introducing selected apelin receptor mutations via CRISPR/Cas-9 gene editing. Conflict of interest None

\section{BS50 PKC $\alpha$ KNOCK-DOWN INCREASES MEDIAL CALCIFICATION IN A MURINE MODEL OF CHRONIC KIDNEY DISEASE}

${ }^{1}$ Samantha Borland, ${ }^{1}$ Cecilia Facchi* ${ }^{1}$ Julia Behnsen, ${ }^{1}$ Antony Adamson, 'Neil Humphrey, ${ }^{1}$ Michael J Sherratt, ${ }^{1}$ Keith Brennan, ${ }^{2}$ Sheila Francis, 'Nick Ashton, ${ }^{1}$ Ann Canfield. ${ }^{1}$ University of Manchester; ${ }^{2}$ University of Sheffield

\subsection{6/heartjnl-2019-BCS.211}

Medial calcification is the formation of mineralised tissue within the smooth muscle layer of the vessel wall, and frequently occurs in patients with chronic kidney disease. Calcification within the medial layer of the vessel wall can reduce aortic and arterial elasticity, which impairs cardiovascular haemodynamics and results in a significantly elevated risk of morbidity and mortality in the form of hypertension, cardiac hypertrophy and sudden cardiac death. Protein kinase $\mathrm{C} \alpha$ (PKC $\alpha$ ) belongs to the PKC family of serine/threonine kinases and we recently discovered that knocking-down PKC $\alpha$ expression increases high phosphate-induced mineral deposition by vascular smooth muscle cells (VSMCs) in vitro. This study tests the hypothesis that PKC $\alpha$ regulates uraemia-induced medial calcification in vivo.

PKC $\alpha-/-$ mice were generated on the calcification-susceptible DBA/2 background (PKC $\alpha-/-)$ using CRISPR/Cas9 technology. To induce uraemia, wild-type DBA/2 and PKC $\alpha-/-$ mice underwent a two-stage sub-total nephrectomy and were fed a high phosphate $(1.5 \%)$ diet for 8 weeks. Renal function was measured by blood urea nitrogen (BUN). Calcification in the ascending aorta/aortic arch and abdominal aorta were analysed and quantified by micro CT and histology. On average, 68.6

$\pm 3 \%(\mathrm{SD}, \mathrm{n}=3)$ of renal mass was removed from wild-type and $65 \pm 3.2 \% \quad(n=5)$ was removed from PKC $\alpha-/-$ mice $(\mathrm{P}>0.05)$. Loss of $\mathrm{PKC} \alpha$ significantly increased uraemiainduced medial calcification in the abdominal aorta $(-20$-fold increase, $\mathrm{P}<0.05)$ when compared to wild-type controls; there is also a trend for calcification to be increased in the ascending aorta/aortic arch of PKC $\alpha-/-$ mice. Whilst there is a trend for BUN levels to be elevated in PKC $\alpha-/-$ mice $(-1.3$-fold increase compared to wild-type controls, $\mathrm{P}=0.1$ ), there is no correlation between BUN levels and the extent of calcification in these mice.

We have shown previously that inhibiting transforming growth factor- $\beta$ (TGF- $\beta$ ) signalling with SB431542 prevents the increase in calcification observed in PKC $\alpha$-siRNA treated VSMCs. Therefore, to determine the mechanism by which loss of PKC $\alpha$ exerts its effects we examined the relationship between PKC $\alpha$ and TGF- $\beta$ signalling in vitro and in vivo. Our results show that knock-down of PKC $\alpha$ using siRNA increased TGF- $\beta 1$-induced Smad2 phosphorylation in VSMCs in vitro $(\mathrm{P}<0.05)$. Furthermore, phosphorylated Smad2 immunostaining was detected throughout calcified aortic arches from PKC $\alpha-/$ - mice. In contrast, only small areas of phosphorylated Smad2 immunostaining was detected in calcified wild-type controls.

In conclusion, our study suggests that $\operatorname{PKC} \alpha$ may play a protective role in uraemia-induced medial calcification. The PKC $\alpha / T G F \beta$ signalling axis could therefore represent a new therapeutic target for uraemia-induced medial calcification. Conflict of interest None

\title{
BS51 AN EVALUATION OF METHODOLOGIES TO INDUCE CALCIFICATION IN VITRO IN HUMAN PRIMARY CELLS
}

Sophie Millar*, Susan Anderson, Saoirse O'Sullivan. University of Nottingham

\subsection{6/heartjnl-2019-BCS.212}

Introduction Vascular calcification (VC), the progressive deposition of calcium within the vasculature, is a major risk factor for morbidity, mortality and cardiovascular disease, particularly within chronic kidney disease (CKD) patients. Accurate and reliable in vitro models are essential to allow for investigation into the complex mechanisms behind this extra-osseous biomineralization process. We aimed to investigate the efficacy of a range of media compositions under various conditions (normoxia and hypoxia) to induce calcification in vitro in human

DMEM mineralisation media

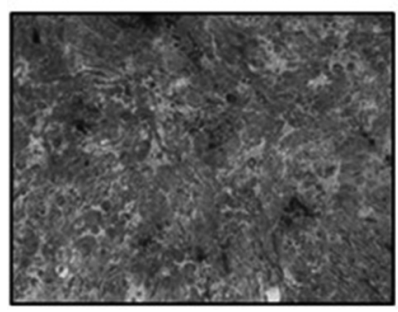

Abstract BS51 Figure 1 Alizarin REd Dye staining after 21 days of culture in DMEM mineralisation media(left) and PromoCell mineralisation media(right) 